\title{
Evaluación del potencial antioxidante en extracto de espinaca por voltamperometría cíclica
}

\author{
Evaluation of antioxidant potential in a spinach \\ extract by cyclic voltammetry
}

\section{Avaliação do potencial antioxidante no extrato de espinafre por voltametria cíclica}

\author{
Susan B. Pérez-Cabeza*; Nicolás Morón Angarita; Martha Cervantes; Mario A. Barón-Rodríguez**. \\ Grupo de Investigaciones Ambientales para el Desarrollo Sostenible. Facultad de Química Ambiental. División de \\ Ingenierías y Arquitectura. Universidad Santo Tomás, carrera 27 N 180-395 Autopista Floridablanca. \\ Bucaramanga, Colombia. \\ "susanbperez@gmail.com; **mbaronro@gmail.com
}

Fecha Recepción: 19 de marzo de 2016 Fecha Aceptación: 16 de agosto de 2017

\begin{abstract}
Resumen
La capacidad antioxidante de extractos de plantas ha sido estudiada por técnicas voltamperométricas gracias a su sensibilidad, selectividad, bajo costo, simplicidad y rapidez. En esta investigación se utilizó la Voltamperometría Cíclica para estudiar el potencial antioxidante en la preservación de extractos etanólicos de hojas de espinaca (Spinacia oleracea). Los extractos se obtuvieron luego de macerar hojas de espinaca con etanol industrial y llevar a ultrasonido. Los barridos potenciométricos de los extractos a 0,7 y 15 días de preparación se realizaron en una solución amortiguadora Sørensen. Se utilizaron electrodos de $\mathrm{Au}$ (trabajo), $\mathrm{Ag} / \mathrm{AgCl}$ (referencia) y $\mathrm{Pt}$ (auxiliar) desde $-1,2$ hasta 1,2V. Los resultados mostraron una alta capacidad antioxidante de los extractos (potenciales de oxidación por debajo de los $600 \mathrm{mV}$ ) y una disminución en la corriente durante el aumento de la preservación de los extractos (0, 7 y 15 días); lo cual permitió concluir que su potencial antioxidante disminuye a través del tiempo gobernada por una cinética de primer orden. Además, los resultados de la capacidad antioxidante fueron correlacionados con la cuantificación de polifenoles totales, mediante en método de Folin Ciocalteu.
\end{abstract}

Palabras clave: radicales libres, estrés oxidativo, voltamperometría cíclica, extracto de espinaca, potencial antioxidante.

\begin{abstract}
The antioxidant capacity of plant extracts has been studied by voltammetric techniques thanks to its sensitivity, selectivity, low cost, simplicity and speed. In this research, Cyclic Voltamperometry was used to study the antioxidant potential and stability in ethanolic extracts of spinach leaves (Spinacia oleracea) over time. The extracts were obtained after ultrasound of macerating spinach leaves with industrial ethanol. The potentiometric sweeps of the extracts at 0,7 and 15 days of preparation were carried out in a Sørensen buffer. Electrodes of $\mathrm{Au}$ (work), $\mathrm{Ag} / \mathrm{AgCl}$ (reference) and $\mathrm{Pt}$ (auxiliary) were used from -1.2 to $1.2 \mathrm{~V}$. The results showed a high antioxidant capacity of the extracts (oxidation potentials below $600 \mathrm{mV}$ ) and a decrease in the current while the extracts preservation (0, 7 and 15 days), which allows the conclusion that its Antioxidant potential decreases over time describing a first-order kinetic. Also, the results of the antioxidant capacity were correlated with the Folin Ciocalteu method.
\end{abstract}

Keywords: free radicals, oxidative stress, cyclic voltammetry, spinach extract, antioxidant potential.

Cita: Pérez-Cabeza SB, Morón Angarita N, Cervantes M, Barón-Rodríguez MA. Evaluación del potencial antioxidante en extracto de espinaca por voltamperometría cíclica. rev.ion. 2017;30(2):99-105. 


\section{Resumo}

A capacidade antioxidante de extratos de plantas foi estudada por meio de técnicas voltamétricas atribuído à sua sensibilidade, seletividade, baixo custo, simplicidade e velocidade. Voltametria cíclica foi utilizada nesta pesquisa para estudar o potencial antioxidante e estabilidade no extrato etanolico de folhas de espinafre (Spinacia oleracea) ao longo do tempo. Esses extratos foram obtidos por maceração das folhas de espinafre com álcool industrial e sonificadas por 40min. Os barridos potenciometricos dos Extratos foram realizados numa solução tampão de Sorensen. Eléctrodos de trabalho $(\mathrm{Au}), \mathrm{de} \mathrm{Ag} / \mathrm{AgCl}$ (referência) e Pt (eléctrodo auxiliar) a uma velocidade de varrimento de $100 \mathrm{mV} / \mathrm{s}$ foram usadas $-1,2$ a $-1,2 \mathrm{mV}$. Os resultados mostraram uma elevada capacidade antioxidante dos extratos (potenciais de oxidação $\mathrm{mV}$ abaixo de 600) e uma diminuição na corrente com respeito à idade dos extratos $(0,7$ e 15 dias), o que permitiu concluir que o potencial antioxidante diminui ao longo do tempo por uma cinética de primeira ordem. Além disso, os resultados da capacidade antioxidante foram correlacionados com a cuantificação de polifenoles totales, através do método de Folin Ciocalteu.

Palavras-chave: radicais livres, estresse oxidativo, voltametria cíclica, extrato de espinafre, potencial antioxidante.

\section{Introducción}

Las frutas y vegetales son excelentes fuentes naturales de antioxidantes como vitaminas, carotenoides, flavonoides y otros compuestos fenólicos [1,2]. Los antioxidantes juegan un papel de gran importancia en la industria alimentaria, cosmética y farmacéutica. Los antioxidantes son compuestos que pueden interactuar con los radicales libres y poner fin a las reacciones en cadena antes que afecten a las moléculas vitales, la eficiencia de este proceso depende de las características químicas del alimento que provee este tipo de compuestos.[3]. Además, son sustancias que buscan retardar el comienzo y disminuir la velocidad de oxidación de las sustancias que presentan características oxidables en nuestro organismo[4].

Diferentes métodos han sido empleados con el fin de estudiar la capacidad antioxidante de extractos naturales de especies vegetales [5,6]: entre los más empleados se encuentran los métodos electroquímicos, que surgen a partir de la década pasada y su fundamento se basa en la capacidad que presentan los antioxidantes para donar electrones [7]. La voltamperometría cíclica (VC), es una técnica electroquímica ampliamente utilizada para la evaluación de la capacidad antioxidante total [8]. Su principal ventaja es la facilidad para determinar rápidamente el comportamiento redox total de mezclas complejas sin la necesidad de medir la capacidad antioxidante de cada componente [9].

Los compuestos polifenólicos de tipo flavonoides, presentes en frutas y verduras, están relacionados con hábitos dietéticos sanos [10]. Dentro de los beneficios que ofrece a la salud consumir este tipo de alimentos se resalta la reducción de enfermedades degenerativas crónicas como las enfermedades cardiovasculares, cáncer y otras [11]. Estos beneficios se encuentran asociados a las propiedades antioxidantes presentes en los alimentos, los cuales han llevado a los investigadores a buscar y entablar un vínculo entre la reducción del riesgo de enfermedades degenerativas y la ingesta de antioxidantes, que se relacionan a la dieta a través de la prevención del daño oxidativo celular [12]. La capacidad antioxidante de estos compuestos, en especial la de los polifenoles; producidos por el metabolismo celular o en respuesta a factores externos, se debe a la captura e inactivación de los radicales libres y especies reactivas del oxígeno (ROS). Este mecanismo de acción previene trastornos degenerativos en los seres humanos, provocados por oxidación de ácidos nucleicos, proteínas y lípidos [13].

La espinaca (Spinacia oleracea) ha sido identificada como un alimento antioxidante de gran valor, además de ser un producto natural de consumo cotidiano $[14,15]$. El alto contenido total de flavonoides en la espinaca $(1.000 \mathrm{mg} / \mathrm{kg})$, en comparación con otras verduras, ofrece numerosas propiedades farmacológicas, antioxidantes, antiinflamatorias, anti-mutagénicas y anticancerígenas $[14,16]$. Con el fin de evaluar la disposición de estos compuestos de alto interés, luego de procesos convencionales de preparación de vegetales, se valoró la capacidad antioxidante y se relacionó con la cantidad de fenoles totales de la espinaca y otras verduras después del proceso de cocción por hervor, vapor o sometimiento a microondas. Los resultados mostraron que procesos moderados de coción no ocasionan eliminación de la capacidad 
antioxidante y fenoles totales de los extractos [17]. Otro factor importante en la disposición de vegetales es su preservación. Es por esto, que se vió la necesidad de evaluar, en tiempo real, el cambio de la capacidad antioxidante de extractos de espinaca preservados durante 15 días y correlacionar estos valores con la cantidad de polifenoles, debida a su conocida capacidad antioxidante.

\section{Parte experimental}

Materiales y reactivos. Para la preparación del extracto se usaron hojas frescas de espinaca (Spinacia oleracea) y el alcohol utilizado fue etanol antiséptico comercial $96 \%$ marca JGB. La solución amortiguadora Sørensen fue preparada con $\mathrm{NaH}_{2} \mathrm{PO}_{4} \cdot 1 \mathrm{H}_{2} \mathrm{O}$ al $98 \%$ pureza marca Merck y $\mathrm{Na}_{2} \mathrm{HPO}_{4} \cdot 7 \mathrm{H}_{2} \mathrm{O}$ al $98 \%$ marca Merck. Para la cuantificación de polifenoles totales se usó Ácido Gálico grado analítico $98 \%$ marca Merck, $\mathrm{Na}_{2} \mathrm{CO}_{3}$ $99 \%$ marca Merck y reactivo Folin-Ciocalteu $2 \mathrm{~N}$ Sigma-Aldrich.

Las hojas de espinacas se pesaron en una balanza analítica Metler Toledo AL 204 (Máx. 210 g-min 0,01g), sensor PT 100. El macerado se realizó en un mortero de porcelana. La extracción por ultrasonido se llevó a cabo en un equipo NEY Ultrasonik. Los voltamogramas cíclicos fueron obtenidos utilizando un potenciostato VersaSTAT 3 conectado a una tarjeta A/D Cassy 524010 y una celda electroquímica modelo KO264 micro cell con un sistema de tres [3] electrodos así: oro (electrodo de trabajo), platino (contra- electrodo) y $\mathrm{Ag} / \mathrm{AgCl} /$ $\mathrm{KCl} 3 \mathrm{M}$ (electrodo de referencia). El programa utilizado para el análisis del comportamiento electroquímico fue el VersaStudio electrochemistry software versión 2.2. Las medidas en ultravioleta Visible para determinación de polifenoles totales se llevaron a cabo en un espectrofotómetro marca Espectroline model ENF-24OC.

Preparación del extracto. Se pesaron $2 \mathrm{~g}$ de hojas de espinacas, se maceraron en un mortero durante 20 minutos; durante el proceso se adicionaron $10 \mathrm{~mL}$ de alcohol etílico. A continuación se tapó la mezcla con papel parafilm y se llevó a ultrasonido durante 40 minutos, para luego realizar un proceso de filtración simple con papel filtro franja azul. El extracto obtenido fue dividido en tres porciones; cada porción fue envasada en un recipiente tipo ámbar.
Preservación de muestras: Dos porciones del extracto preparado fueron preservados por 7 y 15 días; refrigerados a $5^{\circ} \mathrm{C}$ en viales de $3 \mathrm{~mL}$ herméticamente cerrados y protegidos de la luz con papel aluminio. Después de este tiempo se hicieron las respectivas corridas voltamperométricas.

Preparación de la solución Sørensen. Se preparó una solución $0,2 \mathrm{M}$ de $\mathrm{NaH}_{2} \mathrm{PO}_{4} \cdot \mathrm{H}_{2} \mathrm{O}$, otra $0,2 \mathrm{M}$ de $\mathrm{Na}_{2} \mathrm{HPO}_{4} \cdot 7 \mathrm{H}_{2} \mathrm{O}$ y se mezcló en una proporción 23:2. Posteriormente, se diluyó esta solución con agua destilada en una proporción 1:1 para obtener una concentración de $0,1 \mathrm{M}$. Por último se corroboró el pH neutro de la solución.

Barrido por voltamperometría cíclica. Se realizó el barrido en un intervalo de potencial de $-1,2$ a 1,2V y una velocidad de $100 \mathrm{mV} / \mathrm{s}$. Antes de iniciar el proceso se burbujeó Argón durante 3 minutos para desplazar el oxígeno presente en cada muestra a analizar. El electrodo de oro (trabajo) fue pulido antes de cada experimento utilizando una lija $\mathrm{N}^{\circ}$ 1200 , alúmina en polvo de 0,05 micras de Buehler micropolish y Diamond polishig compoud de Buhler. Inicialmente, se tomó un voltamperograma de $2 \mathrm{~mL}$ de la solución amortiguadora; para obtener el blanco. Seguidamente, se tomó la lectura al adicionar $20 \mu \mathrm{L}$ de extracto recién preparado (Muestra fresca) a la solución Sørensen. Este mismo proceso experimental se llevó a cabo con los extractos de espinacas preservados 7 y 15 días.

Cuantificación de polifenoles totales con reactivo de Folin-Ciocalteu. Se preparon una solución madre de ácido gálico $\left(\mathrm{C}_{7} \mathrm{H}_{6} \mathrm{O}_{5}\right)$ a una concentración de $5.000 \mathrm{ppm}$ y una solución de $\mathrm{Na}_{2} \mathrm{CO}_{3}$ al $10 \%$. A continuación se prepararon patrones de 50 a $500 \mathrm{ppm}$ de ácido gálico y se realizó una curva de calibración. A cada patrón se le adicionaron $6,5 \mathrm{~mL}$ de agua destilada y $500 \mu \mathrm{L}$ del reactivo Folin-Ciocalteu; se agitó con vortex y se dejó reposar durante 8 minutos a temperatura ambiente. Transcurrido este tiempo, se adicionaron $3 \mathrm{~mL}$ de carbonato de sodio, se agitó nuevamente con vortex y se dejó en baño María durante 30 minutos a una temperatura de $30^{\circ} \mathrm{C}$. Luego, se realizó la lectura en el espectrofotómetro a una longitud de onda de 765nm [18]. Se usó el mismo procedimiento para la determinación de polifenoles totales en las muestras (extractos). 
Con el objeto de corroborar la reproducibilidad de los resultados, se realizaron tres réplicas de los procedimeintos mencionados.

\section{Resultados y Discusión}

\section{Voltamperometría cíclica (VC)}

Como era de esperarse el voltamperograma de la solución Sørensen (Blanco en Figura 1a) no muestra reacciones redox significativas, lo que indica que constituye una adecuada línea base para la evaluación de la actividad antioxidante y una solución amortiguadora segura para la disolución de las muestras a analizar. Para valorar la autenticidad del método empleado, se validó comparativamente al determinar el voltamperograma cíclico de la vitamina C
(Figura 1b); este voltamperograma muestra el perfil característico de la vitamina $\mathrm{C}$ en solución Sørensen de trabajo [19].

Por otra parte, los voltamperogramas cíclicos de los extractos etanólicos de espinaca en solución Sørensen (Extractos en Figura 1a) evidencian potenciales característicos de soluciones con compuestos antioxidantes [20]. El potencial de oxidación entre $-0,590 \mathrm{~V}$ y $0,339 \mathrm{~V}$, para la muestra fresca, i.e., extracto recién preparado (Extracto 0), sugiere la existencia de reacciones redox típicas de compuestos fenólicos; como antioxidantes [6]. Los voltamperogramas de los extractos guardados durante 7 y 15 días (Extractos 1 y 2 en Figura 1a) muestran comportamientos semejantes: en ambos casos se observa un perfil similar a la primera muestra con respecto al potencial de oxidación.
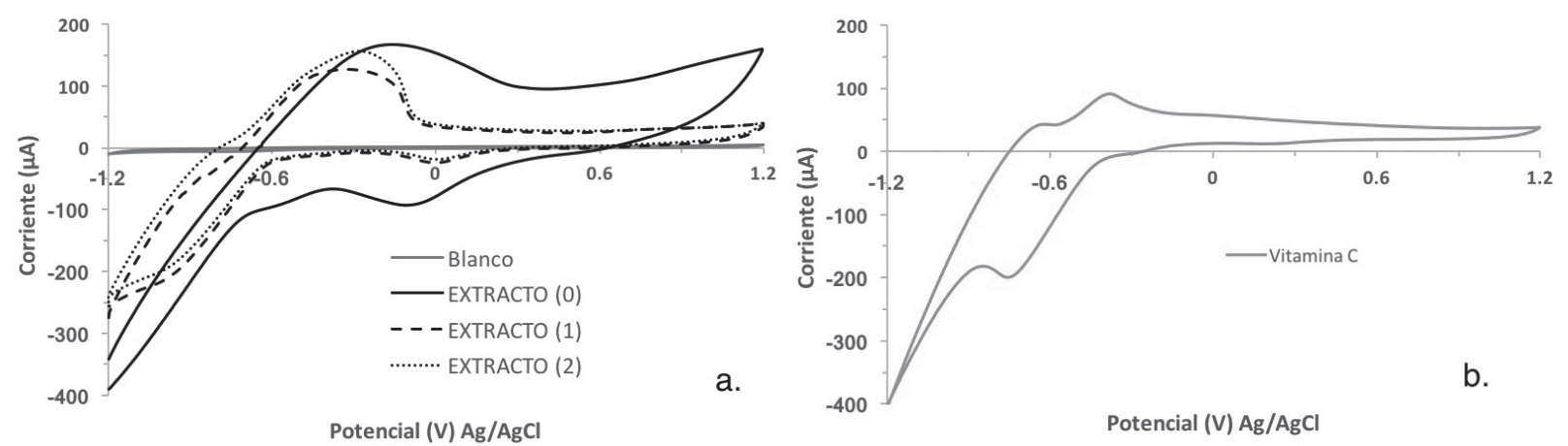

Figura 1. Voltamperogramas cíclicos: a. Extractos de espinaca (Solución Sørensen como blanco, Extracto (0) fresco, Extracto (1) 7 días de preservación, Extracto (2) 15 días de preservación; b. Solución de vitamina C, $50 \mathrm{ppm}$. Todos los barridos fueron realizados en la solución Sørensen a pH 7.

Adicionalmente, se evaluó el corrimiento de los picos de oxidación de los extractos de espinaca en comparación con los de la vitamina $\mathrm{C}$, a condiciones estándar de solución electrolítica Sørensen a pH 7 (Tabla 1). Si bien los extractos de espinaca no presentan un potencial de oxidación igual a la vitamina $C$, sus valores están dentro del rango aceptable para ser clasificados como de excelente actividad antioxidante. Por otra parte, se observa que al pasar el tiempo de almacenamiento de los extractos de espinaca los picos del potencial de oxidación presentan un corrimiento hacia valores más negativos; esto podría sugerir que el potencial de oxidación aumentará durante el proceso de almacenamiento. Sin embargo, el fenómeno que se presenta es que durante el proceso de almacenamiento, los compuestos antioxidantes de menor potencial se oxidan y cumplen su función preservadora del extracto; por esta razón se observan distribuciones no gausianas, principalmente en la parte derecha de la banda, en los voltamperogramas de los extractos almacenados durante 7 y 15 días (Figura 1a).

Tabla 1. Potenciales de oxidación de los extractos de espinaca y la vitamina $C$.

\begin{tabular}{cc}
\hline Muestra & Potencial de Oxidación \\
\hline Extracto (0) & $-0,149 \mathrm{~V}$ \\
Extracto (1) & $-0,248 \mathrm{~V}$ \\
Extracto (2) & $-0,252 \mathrm{~V}$ \\
Vitamina C & $-0347 \mathrm{~V}$ y $-0,592 \mathrm{~V}$ \\
\hline
\end{tabular}

Debido a que el pico de máxima corriente se desplazó hacia potenciales mayores, a medida que aumentaba el tiempo del almacenamiento, se decidió determinar la corriente total de la banda por potencial de oxidación para los extractos; representada como el área bajo la curva del 
potencial de oxidación (Figura 2) [21]. En esta ocasión, se muestra (Tabla 2) que el mayor área se presenta en el extracto fresco, i.e. preparado inmediatamente antes del análisis potenciométrico. Además, se muestra que a medida que transcurre el tiempo de almacenamiento, la capacidad antioxidante total disminuye exponencialemente con una cinética de primer orden (Ecuación 1).
Donde la variable independiente es el tiempo del extracto, en días, y la variable dependiente es el logaritmo natural del área bajo la curva de la relación corriente - potencial de oxidación); debido a que a mayor área bajo la curva (Tabla 2) es mayor la capacidad antioxidante del extracto [4].

$$
\begin{gathered}
y=-0,091 x+4,856 \\
R^{2}=0,999
\end{gathered}
$$

Tabla 2. Área bajo la curva de los picos de oxidación de los extractos de espinaca.

\begin{tabular}{cccc}
\hline Muestra & $\begin{array}{c}\text { Área bajo la curva } \\
(\mathbf{V} \text { x mA) }\end{array}$ & Potencial (V) & Corriente (mA) \\
\hline Extracto (0) & 128,1 & $-0,440-0,339$ & $100,0-96,4$ \\
Extracto (1) & 68,4 & $-0,617--0,082$ & $46,3-45,3$ \\
Extracto (2) & 32,7 & $-0,631--0,082$ & $51,2-53,5$ \\
\hline
\end{tabular}
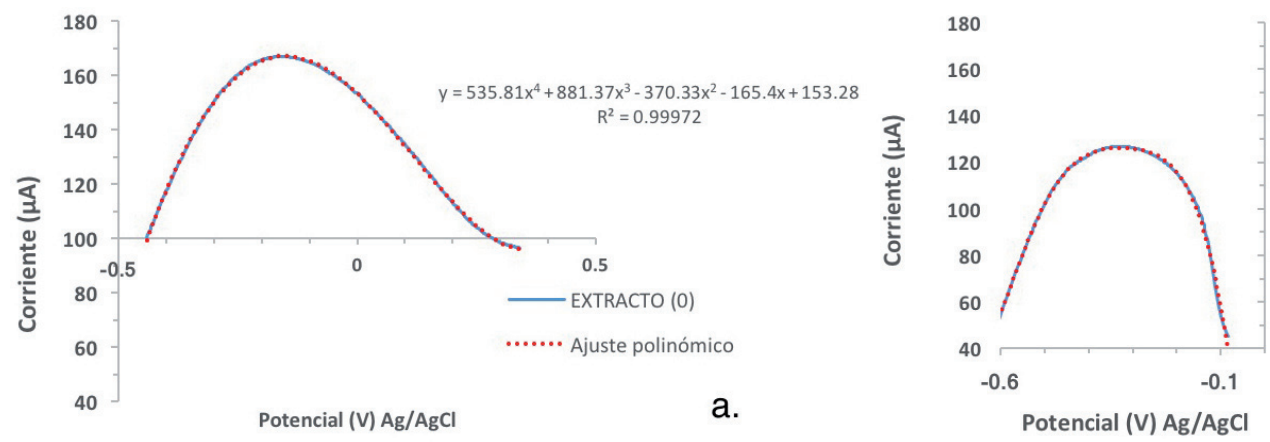

$y=39332 x^{6}+48980 x^{5}+$ $1242.6 x^{4}-22658 x^{3}-13058 x^{2}$ $-3016.4 x-135.3$ (V) Ag/AgCl

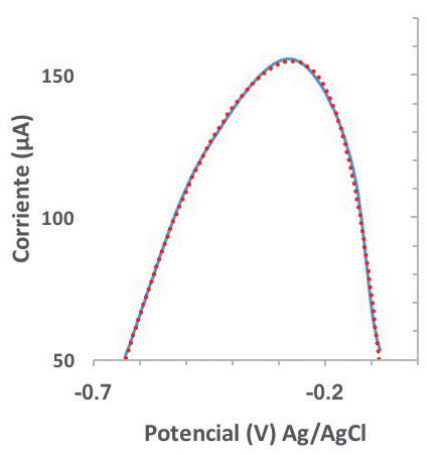

c.

Figura 2. Tendencias polinómicas de los picos de oxidación de los extractos de espinaca; con los cuales se determinó el área bajo la curva. a. Extracto (0); b. Extracto (1); c. Extracto (2).

\section{Polifenoles totales}

A partir de la curva de calibración del ácido gálico, identificado por el método Folin Ciocalteu, y las absorbancias de las muestras, medidas a $765 \mathrm{~nm}$, se estimó la concentración de los polifenoles totales de los extractos etanólicos (Tabla 3) en función de la cantidad de ácido gálico. 
rev.ion. 2017;30(2):99-105. Bucaramanga (Colombia).

Tabla 3. Concentraciones de polifenoles totales en los extractos de espinaca.

\begin{tabular}{ccc}
\hline Muestra & $\begin{array}{c}\text { Concentración de ácido gálico en } \\
\text { extracto }(\mathbf{m g} / \mathbf{L})\end{array}$ & $\begin{array}{c}\text { Concentración de ácido gálico por gramo de } \\
\text { hoja } \mathbf{( m g / g )}\end{array}$ \\
\hline Extracto (0) & 239,4 & 1,195 \\
Extracto (1) & 62,62 & 0,3131 \\
Extracto (2) & 29,21 & 0,1460 \\
\hline
\end{tabular}

$$
\begin{gathered}
y=-0,139 x+5,351 \\
R^{2}=0,962
\end{gathered}
$$

En esta ocasión también se corroboró la disminución exponencial de los polifenoles totales con una cinética de primer orden (Ecuación 2). Donde la variable independiente es el tiempo del extracto, en días, y la variable dependiente es el logaritmo natural de la concentración de polifenoles; medido como concentración de ácido gálico en el extracto), parcialmente responsables de la actividad antioxidante, durante el almacenamiento de los extractos. Al hallar la concentración de polifenoles totales por gramo de hoja fue necesario tener en cuenta la cantidad de disolvente usado en la preparación de los extractos.

Los resultados anteriores (Figura 3, Tabla 3, Ecuación 2) muestran que evidentemente la concentración de polifenoles, responsables de la actividad antioxidante de la espinaca, decrece exponencialmente con una cinética de primer orden a medida que el extracto es más antiguo. Lo cual es coherente con los resultados obtenidos en el análisis voltamperométrico. Sin embargo, la velocidad de degradación de los polifenoles es ligeramente mayor (Ecuación 2) que la velocidad de totalidad de antioxidantes (Ecuación 1); esto podría indicar que los polifenoles tienen más tendencia a ejercer su función antioxidante que los otros antioxidantes de los extractos. Además, estos resultados, muestran con pruebas electroquímicas y espectrométricas la importancia del uso de vegetales frescos en la alimentación y la velocidad de su degradación desde la perspectiva de su potencial antioxidante.

Para esclarecer inquietudes asociadas a la velocidad de oxidación de especies químicas en los estractos, se recomienda en investigaciones futuras usar técnicas de separación de compuestos químicos, como cromatografía líquida de alta eficiencia (HPLC) para determinar la concentración de dichas especies en tiempo real.

\section{Conclusiones}

Los perfiles voltamperográficos de los diferentes extractos de espinaca mostraron potenciales de oxidación entre $-0,252 \mathrm{~V}$ y $-0,149 \mathrm{~V}$; esto sugiere la presencia de al menos un compuesto antioxidante. La comparación de estos perfiles con el voltamperograma de vitamina $C$ revela que los extractos de espinaca tienen una actividad antioxidante muy cercana al patrón de referencia.

Por otra parte, la estimación del área bajo la curva de los picos de oxidación, en los extractos de espinaca, permitió concluir que el tiempo de preservación de los extractos de espinaca ocasiona una disminución de la actividad antioxidante de los mismos; gobernada por una cinética de primer orden.

La correlación entre las cinéticas de disminución de la capacidad antioxidante obtenidos por voltametría cíclica y disminución de los polifenoles totales obtenidos por el método espectrofotométrico durante la preservación de los extractos, ratifica que los resultados obtenidos VC son fiables y comparables con los resultados de otros métodos comúnmente empleados.

Por último, la investigación corroboró la Voltamperometría Cíclica de mezclas complejas como metodología útil para estimar en tiempo real el estado antioxidante de extractos correlación con la presencia de compuestos con poder reductor.

\section{Referencias bibliográficas}

[1] Minussi RC, Rossi M, Bologna L, Cordi L, Rotilio D, Pastore GM, et al. Phenolic compounds and total antioxidant potential of commercial wines. Food Chem. Elsevier. 2003;82(3):409-16.

[2] Zhang D, Hamauzu Y. Phenolics, ascorbic 
acid, carotenoids and antioxidant activity of broccoli and their changes during conventional and microwave cooking. Food Chem. Elsevier. 2004;88(4):503-9.

[3] Oroian M, Escrichel.Antioxidants: Characterization, natural sources, extraction and analysis. Food Res Int. 2015;74:10-36.

[4] La A, Toro R, Fredy G, López V, Taipe M. Evaluación De La Actividad Antioxidante Del Pisco Peruano Mediante Voltametría Cíclica. Rev Soc Quím Perú. 2011;77(2):127-34.

[5] Sochor J, Dobes J, Krystofova O, Ruttkaynedecky B, Babula P, Pohanka M, et al. Electrochemistry as a Tool for Studying Antioxidant Properties. Int. J. Electrochem. Sci. 2013;8:8464-89.

[6] Ruiz-Montoya M, Palma A, Alonso-Garrido G, Pintado S, Chemistry P, Chemistry O, et al. Comparison of the Simple Cyclic Voltammetry (CV) and DPPH Assays for the Determination of Antioxidant Capacity of Active Principles. 2012;5126-38.

[7] Zieliński H, Zielińska D, Kostyra H. Antioxidant capacity of a new crispy type food products determined by updated analytical strategies. Food Chem. 2012;130(4):1098-104.

[8] Simić A, Manojlović D, Segan D, Todorović M. Electrochemical behavior and antioxidant and prooxidant activity of natural phenolics. Molecules. 2007;12(10):2327-40.

[9] Brcanović JM, Pavlović AN, Mitić SS, Stojanović GS, Manojlović DD, Kaličanin $\mathrm{BM}$, et al. Cyclic voltammetry determination of antioxidant capacity of cocoa powder, dark chocolate and milk chocolate samples: Correlation with spectrophotometric assays and individual phenolic compounds. Food Technol Biotechnol. 2013;51(4):460-70.

[10] Piovesan JV, Jost CL, Spinelli A. Electroanalytical determination of total phenolic compounds by square-wave voltammetry using a poly(vinylpyrrolidone)-modified carbon-paste electrode. Sensors Actuators B Chem. 2015;216:192-7.

[11] García E, Fernandez I, Fuentes A. Determinación de polifenoles totales por el método de Folin- Ciocalteu. Univ Politécnica Val; 2015. http://hdl.handle.net/10251/52056.

[12]Bernaert N, De Paepe D, Bouten C, De Clercq $\mathrm{H}$, Stewart $\mathrm{D}$, Van Bockstaele $\mathrm{E}$, et al. Antioxidant capacity, total phenolic and ascorbate content as a function of the genetic diversity of leek (Allium ampeloprasum var. porrum). Food Chem. 2012;134(2):669-77.

[13] Arteaga JF, Ruiz-Montoya M, Palma A, AlonsoGarrido G, Pintado S, Rodríguez-Mellad JM. Comparison of the simple cyclic voltammetry (CV) and DPPH assays for the determination of antioxidant capacity of active principles. Molecules. 2012;17(5):5126-38.

[14]Vázquez E, García-Risco MR, Jaime L, Reglero G, Fornari T. Simultaneous extraction of rosemary and spinach leaves and its effect on the antioxidant activity of products. J Supercrit Fluids. 2013;82:138-45.

[15] Howard LR, Pandjaitan N, Morelock T, Gil MI. Antioxidant Capacity and Phenolic Content of Spinach As Affected by Genetics and Growing Season. J. Agric. Food Chem. 2002;50(21):5891-6.

[16] Pandjaitan N, Howard LR, Morelock T, Gil MI. Antioxidant capacity and phenolic content of spinach as affected by genetics and maturation. J. Agric. Food Chem. 2005;53(22):8618-23.

[17] Turkmen N, Sari F, Velioglu YS. The effect of cooking methods on total phenolics and antioxidant activity of selected green vegetables. Food Chem. 2005;93(4):713-8.

[18]Blainski A, Lopes GC, de Mello JC. Application and Analysis of the Folin Ciocalteu Method for the Determination of the Total Phenolic Content from Limonium Brasiliense L. Molecules. 2013;18(6):6852-65.

[19] Arroyo Currás N. Sistema aprótico para el estudio voltamperométrico de polifenoles: actividad antioxidante vs conducta electroquímica (Proyecto de pregrado). Monterrey, México: Instituto Tecnológico y de Estudios Superiores de Monterrey; 2009.

[20] La Rosa Toro A, Vigo López F, Muedas Taipe G. Assessment of the antioxidant activity of peruvian pisco through cyclic voltammetry. Rev. Soc. Quim. Perú. 2011;77(2):127-34.

[21] Chevion S, Chevion M, Chock PB, Beecher GR. Antioxidant capacity of edible plants: Extraction protocol and direct evaluation by cyclic voltammetry. J Med Food. 1999;2(1):1-10. 